\title{
Interaction sites of DivIVA and RodA from Corynebacterium glutamicum
}

\section{Boris Sieger and Marc Bramkamp*}

Biocenter - Ludwig-Maximilians-University Munich, Munich, Germany

\section{Edited by:}

Conrad L. Woldringh, University of Amsterdam, Netherlands (retired)

\section{Reviewed by:}

Sven Halbedel, Robert Koch Institute, Germany

Kevin D. Young, University of

Arkansas for Medical Science, USA

\section{*Correspondence:}

Marc Bramkamp, Biocenter Ludwig-Maximilians-University Munich, Großhaderner Straße 2-4, 82152 Planegg-Martinsried, Munich, Germany

e-mail: marc.bramkamp@Imu.de
Elongation growth in actinobacteria is localized at the cell poles. This is in contrast to many classical model organisms where insertion of new cell wall material is localized around the lateral site. We previously described a role of RodA from Corynebacterium glutamicum in apical cell growth and morphogenesis. Deletion of rodA had drastic effects on morphology and growth, likely a result from misregulation of penicillin-binding proteins and cell wall precursor delivery. We identified the interaction of RodA with the polar scaffold protein DivIVA, thus explaining subcellular localization of RodA to the cell poles. In this study, we describe this interaction in detail and map the interaction sites of DivIVA and RodA. A single amino acid residue in the N-terminal domain of DivIVA was found to be crucial for the interaction with RodA. The interaction site of RodA was mapped to its cytoplasmic, $\mathrm{C}$-terminal domain, in a region encompassing the last 10 amino acids (AAs). Deletion of these 10 AAs significantly decreased the interaction efficiency with DivIVA. Our results corroborate the interaction of DivIVA and RodA, underscoring the important role of DivIVA as a spatial organizer of the elongation machinery in Corynebacterineae.

Keywords: DivIVA, RodA, Corynebacterium glutamicum, protein-protein interactions, polar cell growth, FRET

\section{INTRODUCTION}

Corynebacterium glutamicum is a fast growing, facultative anaerobic, Gram positive Actinobacterium with high industrial importance in the production of amino acids (AAs; Ikeda and Nakagawa, 2003). For this purpose, fast growth rates and high cell densities are two major properties to ensure efficient production rates. Furthermore, C. glutamicum gained medical interest due to its mycobacteria-like cell wall and its phylogenetic relation to notorious pathogens such as C. diphtheriae, Mycobacterium tuberculosis, and M. leprae (Barh et al., 2011; Cayabyab et al., 2012). Members of the genus Corynebacterium are abundant species on the human skin and airways microbiome (Cowling and Hall, 1993; Zeeuwen et al., 2013). C. glutamicum and all other actinobacteria grow apically by insertion of new cell wall material at the cell poles (Locci and Schaal, 1980; Brown et al., 2011; Donovan and Bramkamp, 2014). Spatial localization of the cell wall machinery is governed by a coiled-coil protein, DivIVA (Letek et al., 2008; Sieger et al., 2013). This is in remarkable contrast to rod-shaped bacteria from other phyla such as firmicutes or proteobacteria. In these organisms a MreBCD-based cell wall synthetic machinery is acting along the lateral sites of the cell (Jones et al., 2001; Kruse et al., 2005). The processive enzymes, e.g. penicillin-binding proteins are, however, ubiquitous (Popham and Young, 2003). Furthermore, membrane integral proteins of the SEDS family are found in every cell wall synthetic cluster (Henriques et al., 1998; Pastoret et al., 2004). It is believed that an FtsW homolog is associated with septal cell wall synthesis, while RodA homologs are part of the elongation machinery (Pastoret et al., 2004; Real et al., 2008; Sieger et al., 2013). FtsW and RodA are associated with flipping the cell wall precursor lipid II (Ikeda and
Nakagawa, 2003; Noirclerc-Savoye et al., 2003; Mohammadi et al., 2011). A new candidate for lipid II flipping, MurJ, has recently been described (Sham et al., 2014). Maybe, both enzymes may confer translocation of lipid II. However, based on earlier studies with $\operatorname{rodA}$ deletion mutants in $C$. glutamicum, it became evident that lack of RodA may also influence activity of the cognitive penicillin-binding proteins (Sieger et al., 2013).

In a previous study we identified RodA as being essential for growth and determination of cell shape (Sieger et al., 2013). DivIVA is frequently present in Gram positive species and generally composed of a highly conserved N-terminal domain, followed by two coiled-coil domains (Letek et al., 2009; Figures 1A,B). The N-terminal domain is involved in membrane attachment via exposed phenylalanine residues, positioned at the tip of intertwined loops as revealed by the crystal structure of the Bacillus subtilis DivIVA (Oliva et al., 2010). The coiled-coil domains are required for oligomerization and scaffold formation (Stahlberg et al., 2004; Lenarcic et al., 2009). DivIVA proteins lack an enzymatic function and polymerize into large oligomers in a nucleotide independent fashion (Muchova et al., 2002).

In the past decade several interaction partners of DivIVA have been identified. B. subtilis DivIVA anchors the Min system via MinJ to the cell poles, thus contributing to division site selection (Bramkamp et al., 2008; Patrick and Kearns, 2008). In addition, DivIVA interacts with RacA to attach the DNA to the prespore pole during B. subtilis sporulation (Ben-Yehuda et al., 2003; Wu and Errington, 2003). Further interaction partners are the division inhibitor Maf in competent B. subtilis cells (Briley et al., 2011), the transcriptional regulator ComN during promotion of natural competence (dos Santos et al., 2012) and SpoIIE for asymmetric 


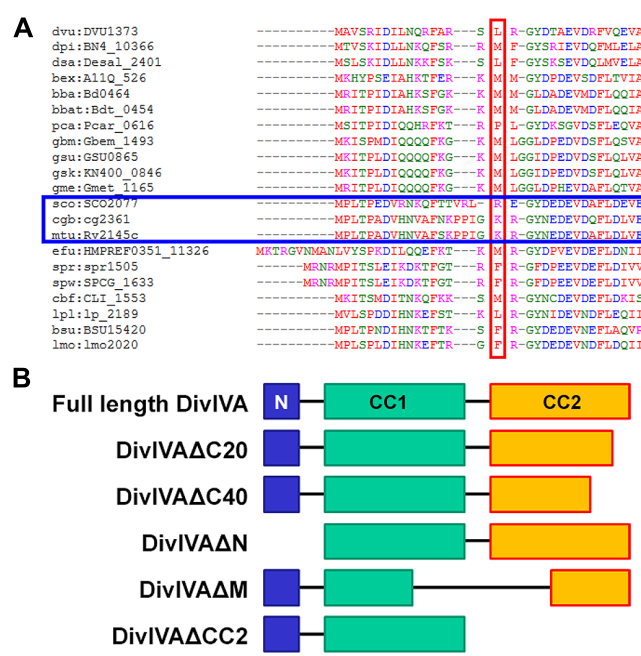

C

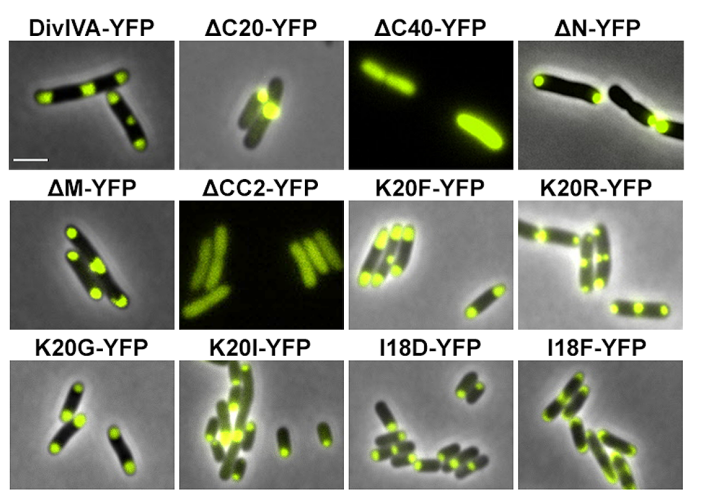

D

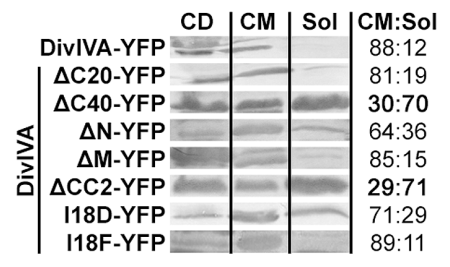

FIGURE 1 | (A) Sequence alignment of $\mathrm{N}$-terminal domains of several DivIVA proteins. Marked in red are AA residues that correspond to the F17 residue located at the tip of intertwined loops, according to the Bacillus subtilis DivIVA crystal structure. Polar growing actinobacteria such as Streptomyces, Corynebacterium, and Mycobacteria have a positively charged residue at this position (marked with a blue box). In the case of $C$. glutamicum, the corresponding AA is the lysine K20. Dvu: Desulfovibrio vulgaris, Dpi: Desulfovibrio piezophilus, Dsa: Desulfovibrio salexigens, Bex: Bdellovibrio exovorus, Bba: Bdellovibrio bacteriovorus, Bbat: Bdellovibrio bacteriovorus Tiberius, Pca: Pelobacter carbinolicus, Gbm: Geobacter bemidjiensis, Gsu: G. sulfurreducens PCA, Gsk: G. sulfurreducens KN400, Gme: G. metallireducens, Sco: Streptomyces coelicolor, Cgb:

Corynebacterium glutamicum, Mtu: Mycobacterium tuberculosis, Efu: Enterococcus faecium DO, Spr: Streptococcus pneumoniae R6, Spw: S. pneumoniae CGSP14, Cbf: Clostridium botulinum F, Lpl: Lactobacillus plantarum WCFS1, Bsu: B. subtilis, Lmo: Listeria monocytogenes. Sequences were obtained from KEGG database. (B) Topology models of full length DivIVA and several truncation mutants that were generated and used in this study. DivIVA $\triangle \mathrm{C} 20$ and DivIVA $\Delta \mathrm{C} 40$ lack 20 or 40 AAs of their $\mathrm{C}$-terminal ends. DivIVA $\Delta \mathrm{N}$ lacks the $\mathrm{N}$-terminal domain, DivIVA $\Delta \mathrm{M}$ lacks a middle part of 154 AAs (144-298) and DivIVACC2 lacks the second coiled coil domain. (C) Fluorescence microscopy images of DivIVA variants that were heterologously expressed in Escherichia coli. Full length DivIVA-YFP

(Continued)

\section{FIGURE 1 | Continued}

localizes to both cell poles and the septum. DivIVA $\Delta$ C20-YFP lost its proper localization character and forms huge, presumably non-functional, aggregates at mostly one cell pole. DivIVA $\triangle$ C40-YFP as well as DivIVACC2-YFP localize in the cytoplasm, likely due to misfolding or lack of oligomerization. DivIVA $\triangle$ N-YFP localizes to the poles; however, not always to both cell poles. DivIVA $\triangle M$ MFFP localizes similar to wild type DivIVA. The DivIVA point mutants K20F and K20R localize similar compared to wild type protein. Mutants K20G-YFP and K20I-YFP showed polar localization, partly to one pole, comparable to DivIVA $\Delta$ N-YFP. Mutant I18D-YFP showed no alterations in localization and membrane binding, whereas mutant I18F-YFP gives rise to increased membrane association. 118 is likely involved in membrane binding due to its hydrophobic character. (D) Ratios of non-aggregated, membrane attached vs. soluble DivIVA mutants. Full length DivIVA-YFP is $88 \%$ membrane attached and similar values were obtained from DivIVA $\triangle$ C20-YFP and DivIVA $\Delta$ M-YFP. DivIVA $\Delta N-Y F P$ is $64 \%$ membrane attached, implicating defects in membrane attachment that were drastically reduced for DivIVA $\triangle$ C40-YFP and DivIVACC2-YFP to 30\% and $29 \%$, respectively. Mutant I18D-YFP had a minor decrease in membrane binding (71\%) whereas I18F-YFP had a similar membrane affinity compared to WT DivIVA-YFP (89\%). CD, cell debris fraction; CM, cell membrane fraction; Sol, soluble fraction. All numbers are mean values of at least three independent experiments.

division during sporulation (Eswaramoorthy et al., 2014). Only recently, it was demonstrated how MinJ and RacA of B. subtilis bind to separate domains of DivIVA (van Baarle et al., 2013). RacA interacts with the $11 \mathrm{C}$-terminal AAs of DivIVA, whereas MinJ binds to the N-terminal lipid binding domain. Although many more interaction partners of DivIVA have been identified in several organisms, as reviewed by (Lin and Thanbichler, 2013; Laloux and Jacobs-Wagner, 2014), little is known about their interaction sites involved in protein-protein interaction.

Here we describe the identification of the RodA-DivIVA interaction sites, thereby corroborating earlier results that suggested an interaction of C. glutamicum DivIVA and RodA (Sieger et al., 2013). Mutational analysis and subsequent interaction studies using a Förster-Resonance Energy Transfer (FRET)-based assay reveals that DivIVA interacts with the C-terminal domain of RodA. The N-terminal domain, which is supposed to play a role in membrane association, is crucial for the interaction with RodA. The molecular mechanism seems to include electrostatic interactions, since a positive charge in DivIVA is essential for full protein-protein interaction.

\section{EXPERIMENTAL PROCEDURES GENERAL CLONING TECHNIOUES}

General cloning was performed as described before (Sieger et al., 2013). Oligonucleotides were obtained from Sigma Aldrich and are listed in Table S1. Plasmids generated in this study are listed in Table 1. C. glutamicum strains are listed in Table 2. Escherichia coli strains that were transformed with the plasmids from Table 1 for cloning and protein expression are not listed separately. Point mutants of DivIVA were generated via overlapping PCR using oligonucleotides carrying the desired point mutation. E. coli was grown in Luria Broth supplemented with $100 \mathrm{mg} \mathrm{ml}^{-1}$ carbenicillin (pETDuet-1) or $50 \mathrm{mg} \mathrm{ml}^{-1}$ kanamycin (pEKEX2). C. glutamicum was grown in Brain Heart Infusion (BHI; Oxoid) supplemented with $25 \mathrm{mg} \mathrm{ml}^{-1}$ kanamycin (pEKEX2). Protein expression was induced with $0.1 \mathrm{mM}$ IPTG. 
Table 1 | Plasmids.

\begin{tabular}{|c|c|c|c|}
\hline No & Name & Description & Reference \\
\hline EX001 & pETDuet-1 & bla, PT7lacl-, PT7lacl- & Novagen \\
\hline BS001 & Duet CFP & bla, PT7lacl-eCFP, PT7lacl- & Sieger et al. (2013) \\
\hline BS002 & Duet YFP & bla, PT7lacl-, PT7lacl-eYFP & Sieger et al. (2013) \\
\hline BS003 & Duet CFPYFP & bla, PT7lacl-eCFP, PT7lacl-eYFP & Sieger et al. (2013) \\
\hline BS005 & Duet RodA-CFP & bla, PT7lacl-rodA-eCFP, PT7lacl- & Sieger et al. (2013) \\
\hline BS006 & Duet FtsW-CFP & bla, PT7lacl-ftsW-eCFP, PT7lacl- & Sieger et al. (2013) \\
\hline BS007 & Duet RodA-CFP DivIVA-YFP & bla, PT7lacl-rodA-eCFP, PT7lacl-divIVA-eYFP & Sieger et al. (2013) \\
\hline BS008 & Duet FtsW-CFP DivIVA-YFP & bla, PT7lacl-ftsW-eCFP, PT7lacl-divIVA-eYFP & Sieger et al. (2013) \\
\hline BS025 & Duet RodA $\Delta$ C10-CFP & bla, PT7lacl-rodA $\triangle$ C10-eCFP PT7lacl- & This study \\
\hline BS029 & Duet RodA $\triangle$ C10-CFP DivIVA-YFP & bla, PT7lacl-rodA $\triangle$ C10-eCFP, PT7lacl-divIVA-eYFP & This study \\
\hline BS030 & Duet RodA $\triangle$ C80-CFP DivIVA-YFP & bla, PT7lacl-rodA $\triangle$ C80-eCFP, PT7lacl-divIVA-eYFP & This study \\
\hline BS031 & Duet RodA1/2-CFP DivIVA-YFP & bla, PT7lacl-rodA1/2-eCFP, PT7lacl-divIVA-eYFP & This study \\
\hline BS032 & Duet RodA2/2-CFP DivIVA-YFP & bla, PT7lacl-rodA2/2-eCFP, PT7lacl-divIVA-eYFP & This study \\
\hline BS033 & Duet DivIVA $\Delta$ C20-YFP & bla, PT7lacl-, PT7lacl-divIVA $\triangle$ C20-eYFP & This study \\
\hline BS034 & Duet DivIVA $\triangle$ C40-YFP & bla, PT7lacl-, PT7lacl-divIVA $\triangle$ C40-eYFP & This study \\
\hline BS035 & Duet DivIVA $\Delta M-Y F P$ & bla, PT7lacl-, PT7lacl-divIVA $\triangle$ M-eYFP & This study \\
\hline BS036 & Duet DivIVA $\Delta N-Y F P$ & bla, PT7lacl-, PT7lacl-divIVA $\Delta$ N-eYFP & This study \\
\hline BS037 & Duet DivIVA $\Delta$ CC2-YFP & bla, PT7lacl-, PT7lacl-divIVA $\triangle$ CC2-eYFP & This study \\
\hline BS044 & Duet RodAE438G-CFP DivIVA-YFP & bla, PT7lacl-rodAE438G-eCFP, PT7lacl-divIVA-eYFP & This study \\
\hline BS045 & Duet RodAK434G-CFP & bla, PT7lacl-rodAK434G-eCFP, PT7lacl- & This study \\
\hline BS046 & Duet RodAK434G-CFP, DivIVA-YFP & bla, PT7lacl-rodAK434G-eCFP, PT7lacl-divIVA-eYFP & This study \\
\hline BS047 & Duet RodAQ435G-CFP & bla, PT7lacl-rodAQ435G-eCFP, PT7lacl- & This study \\
\hline BS048 & Duet RodAQ435G-CFP, DivIVA-YFP & bla, PT7lacl-rodAQ435G-eCFP, PT7lacl-divIVA-eYFP & This study \\
\hline BS049 & Duet RodAS433G-S437G-CFP & bla, PT7lacl-rodAS433G-S437G-eCFP, PT7lacl- & This study \\
\hline BS050 & Duet RodAS433G-S437G-CFP, DivIVA-YFP & bla, PT7lacl-rodAS433G S437G-eCFP, PT7lacl-divIVA-eYFP & This study \\
\hline BS051 & Duet RodAmutC10-CFP & bla, PT7lacl-mutC10-eCFP, PT7lacl- & This study \\
\hline BS052 & Duet RodAmutC10-CFP, DivIVA-YFP & bla, PT7lacl-mutC10-eCFP, PT7lacl-divIVA-eYFP & This study \\
\hline BS053 & Duet DivIVAK20F-YFP & bla, PT7lacl-, PT7lacl-divIVAK2OF-eYFP & This study \\
\hline BS054 & Duet RodA-CFP, DivIVAK20F-YFP & bla, PT7lacl-rodA-eCFP, PT7lacl-divIVAK2OF-eYFP & This study \\
\hline BS055 & Duet DivIVAK20R-YFP & bla, PT7lacl-, PT7lacl-divIVAK20R-eYFP & This study \\
\hline BS056 & Duet RodA-CFP, DivIVAK20R-YFP & bla, PT7lacl-rodA-eCFP, PT7lacl-divIVAK20R-eYFP & This study \\
\hline BS057 & Duet DivIVAK20G-YFP & bla, PT7lacl-, PT7lacl-divIVAK20G-eYFP & This study \\
\hline BS058 & Duet RodA-CFP, DivIVAK20G-YFP & bla, PT7lacl-rodA-eCFP, PT7lacl-divIVAK20G-eYFP & This study \\
\hline
\end{tabular}


Table 1 | Continued

\begin{tabular}{|c|c|c|c|}
\hline No. & Name & Description & Reference \\
\hline BS059 & Duet DivIVAK20I-YFP & bla, PT7lacl-, PT7lacl-divIVAK20l-eYFP & This study \\
\hline BS060 & Duet RodA-CFP, DivIVAK20I-YFP & bla, PT7lacl-rodA-eCFP, PT7lacl-divIVAK20l-eYFP & This study \\
\hline BS061 & Duet DivIVAI18D-YFP & bla, PT7lacl-, PT7lacl-divIVA/18D-eYFP & This study \\
\hline BS062 & Duet RodA-CFP, DivIVAI18D-YFP & bla, PT7lacl-rodA-eCFP, PT7lacl-divIVAl18D-eYFP & This study \\
\hline BS063 & Duet DivIVAl18F-YFP & bla, PT7lacl-, PT7lacl-divIVAl18F-eYFP & This study \\
\hline & pEKEX2 & & \\
\hline EX010 & pEXEK2 & $\begin{array}{l}\text { Escherichia coli - C. glutamicum shuttle vector, } \mathrm{Kan}^{\mathrm{R}}, \mathrm{P}_{\text {tac }} \text { lacla, } \\
\text { pBL1 oriV C.g., pUC18 oriV E.c., }\end{array}$ & Eikmanns et al. (1991) \\
\hline BS018 & pEX2 RodA-GFP & $\mathrm{Kan}^{\mathrm{R}}, \mathrm{P}_{\mathrm{tac}} \mid \mathrm{lac} /^{q}, \mathrm{pBL1}$ oriV C.g., pUC18 oriV E.c., rodA-gfp & Sieger et al. (2013) \\
\hline BS053 & pEX2 RodA1/2-GFP & $\operatorname{Kan}^{\mathrm{R}}, \mathrm{P}_{\text {tac }}$ lacl ${ }^{\mathrm{q}}, \mathrm{pBL1}$ oriV C.g., $\mathrm{pUC18}$ oriV $_{E . c .}, \operatorname{rodA1/2-gfp}$ & This study \\
\hline
\end{tabular}

Table 2 | Strains.

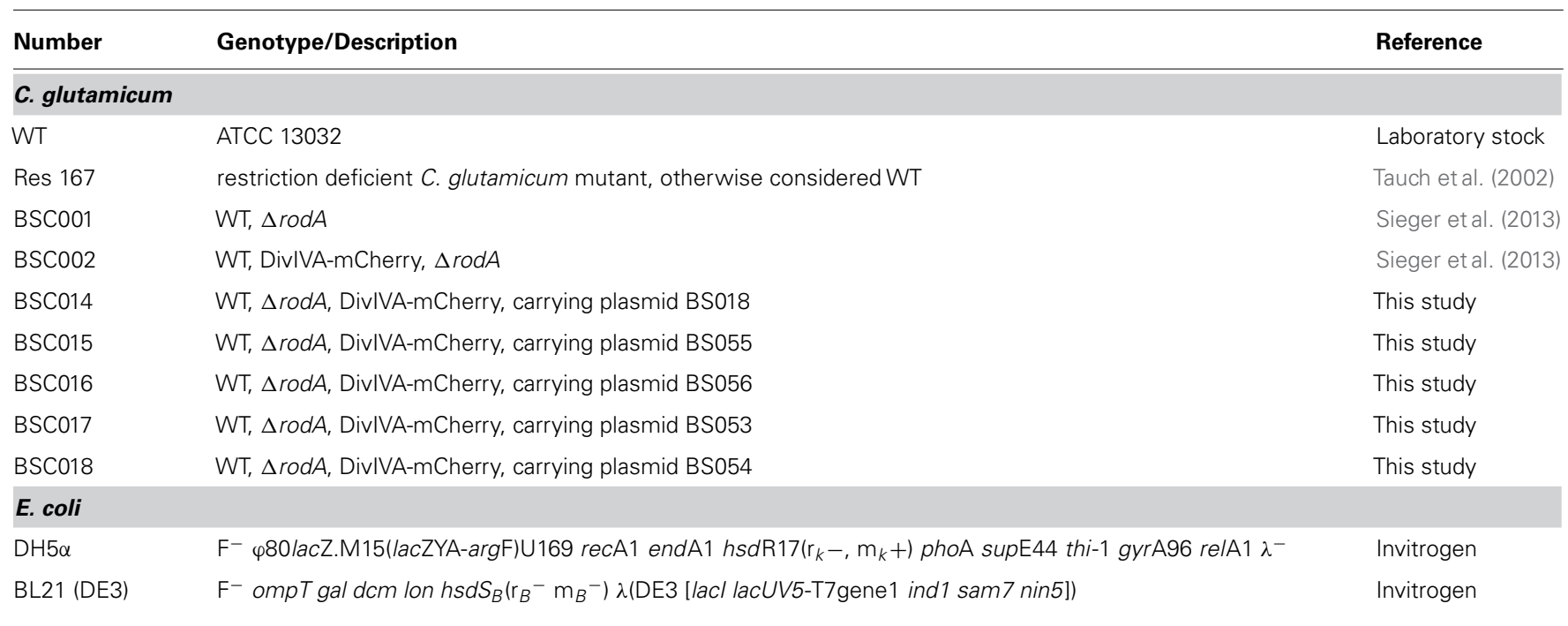

\section{MICROSCOPY}

Microscopy was performed on a Zeiss Axio Observer Z1 microscope equipped with a Hamamatsu OrcaR ${ }^{2}$ camera. A PlanApochromat 100x/1.4 Oil Ph3 objective (Zeiss) was used. YFP fluorescence was visualized with filter set 46 HE YFP shift free and CFP fluorescence with filter set 47 HE CFP shift free (Zeiss). Images were acquired with Zen software (Zeiss) or AxioVision 4.6 (Zeiss) and processed with Adobe Photoshop.

\section{FRET}

Quantitative FRET values $\left(\mathrm{R}_{\mathrm{CY}}\right)$ were calculated as ratios from emission maxima of eCFP (480 nm) and eYFP (525 nm). FRET measurements were performed in late exponential growing $E$. coli cells after one washing step with $0.9 \% \mathrm{NaCl}$. $150 \mu \mathrm{l}$ of cell suspension were loaded into a 96 well microtiter plate and subsequently measured in a Tecan Infinite M200 Pro plate reader. The excitation wavelength was $435 \mathrm{~nm}$; emission was monitored in a range from 466 to $610 \mathrm{~nm}$ in $3 \mathrm{~nm}$ increments.

\section{CELL FRACTIONATION}

Analysis of protein localization was performed by cell fragmentation and subsequent centrifugation. Cells were lysed in a FastPrep homogenizer (MP) in five rounds at $5 \mathrm{~ms}^{-1}$. Cell debris and aggregated proteins were removed by centrifugation at $14000 \times g$ for $20 \mathrm{~min}$ and cell membranes were harvested at $90000 \times g$ for $30 \mathrm{~min}$. Together with the supernatant, samples were run on SDS PAGE and analyzed by immune-blotting. DivIVA-YFP mutants were blotted with an $\alpha$-GFP antibody 
and visualized using an $\alpha$-rabbit-alkaline phosphatase secondary antibody.

\section{RESULTS \\ TRUNCATION MUTANTS OF DivIVA REVEAL DISTINCT DOMAIN FUNCTIONS}

Several truncation mutants of DivIVA were heterologously expressed to analyze importance of the individual domains for protein localization and protein-protein interactions. C. glutamicum DivIVA is composed of a short $\mathrm{N}$-terminal domain $(\mathrm{N})$ and two coiled-coil domains (CC1 and CC2; Figure 1B). The topology and subcellular localization of the mutants are shown in Figure 1C. It turned out that CC2 is responsible for proper folding or assembly of DivIVA oligomers, as deletion of $20 \mathrm{AAs}(\Delta \mathrm{C} 20)$ resulted in aggregation of a likely non-functional protein and deletion of 40 AAs $(\triangle C 40)$ resulted in cytoplasmic appearance. Since CC2 has been reported to play a role in oligomerization in the B. subtilis DivIVA (Oliva et al., 2010), this would explain how DivIVA monomers lose their ability to localize to the cell poles. Deletion of the N-terminal domain had only a minor effect, where some cell poles were free of protein, likely due to reduced membrane attachment (Figure 1D). $64 \%$ of the DivIVA variant $\Delta \mathrm{N}$ was still membrane associated in contrast to $88 \%$ of the wild type protein. Deletion of a middle part of DivIVA ( $\Delta \mathrm{M}$, AA 144-298) had no effect on DivIVA localization and deletion of CC2 showed the same localization defect as $\Delta \mathrm{C} 40$. Cell lysate fractionation confirmed the observation that DivIVA- $\Delta C 40$ and DivIVA- $\Delta C C 2$ fail to localize to the membrane. In these two mutants only $30 \%$ $(\Delta \mathrm{C} 40) / 29 \%(\Delta \mathrm{CC} 2)$ of non-aggregated protein remains membrane attached. Full length DivIVA-YFP, $\Delta \mathrm{C} 20$, and $\Delta \mathrm{M}$ are $88 \% / 81 \% / 85 \%$, membrane associated (Figure 1D).

Data derived from the crystal structure of the B. subtilis DivIVA suggested a role for phenylalanine (F17, B. subtilis numbering) in membrane-binding. This residue is not conserved in most other species (Figure 1A). Members of the class Actinobacteria possess a positively charged residue (arginine, lysine) at the corresponding position (K20 in C. glutamicum and M. tuberculosis). Sequence alignments between various DivIVA sequences reveal that the actinobacterial DivIVA homologs contain sequence insertions compared to other DivIVA sequences and it may be that other hydrophobic AAs such as isoleucine at position 18 (C. glutamicum numbering) might fulfill a function analogous to the F17 from $B$. subtilis (Figure 1A). To approach this idea, I18 was mutated to an aspartate and a phenylalanine and both mutants were tested for membrane attachment. It turned out that I18D revealed a slight decrease in membrane binding $(71 \%)$, whereas I18F had a similar membrane affinity compared to WT (89\%; Figure 1D). Finally, we mutated the lysine residue situated at position 20 in C. glutamicum to analyze influence of localization and DivIVA-RodA interaction. The DivIVA variants K20F and K20R localized properly at the poles and septa (Figure 1C), suggesting that K20 is not essential for membrane binding.

\section{PROTEIN-PROTEIN INTERACTIONS MEASURED BY FÖRSTER- RESONANCE ENERGY TRANSFER (FRET)}

We established a FRET assay which allows visualization of proteinprotein interaction in cell cultures after heterologous expression in E. coli. The proteins of interest are fused to CFP or YFP, respectively and expressed from plasmid pET-Duetl (Novagen), which harbors two multiple cloning sites with two individual T7 promoters. Table 3 shows ratios of emission maxima measured for several strains. Ratios were calculated by dividing emission maxima for CFP $(525 \mathrm{~nm})$ and YFP (480 nm), respectively; thereby giving a $R_{C Y}$ value that allows to judge about putative proteinprotein interactions. We grouped the ratios into steps as indicated in Table 3, which illustrate the interaction situation of several proteins analyzed in vivo. $\mathrm{R}_{\mathrm{CY}}$ values below 0.9 reflect a high CFP emission and low YFP emission, as obtained from strains expressing CFP or CFP-tagged proteins alone. A strain expressing soluble CFP and YFP also falls in this $\mathrm{R}_{\mathrm{CY}}$ range (0.64) and demonstrates the reliability of the assay (Figure S1). Both fluorophores are evenly distributed in the cytosol; however, a FRET signal is not generated even under conditions of extreme overexpression. Values between 0.9 and 1.1 occur when CFP and YFP fluorescent fusions are present in the cell, where some of the fluorescence energy can be transferred from donor to acceptor upon random approximation of the fluorophores. This approximation can be due to topological circumstances, as it is the case for DivIVA and FtsW, a divisome specific RodA homolog. Similar ratios were obtained from other non-interacting examples such as DivIVA with BetP (a betaine carrier) or several RodA and DivIVA mutants (Table 3). In addition, a FRET-based ATP-sensor protein expressed from plasmid pRSETB AT1.03 (Imamura et al., 2009) served as a positive control. The ATP-sensor gave a strong FRET signal under physiological ATP levels $\left(R_{\mathrm{CY}}=1.25\right)$. However, when the pRSETB AT1.03 cells were treated with CCCP to depolarize the membrane and thus reduce the ATP level in the cell, the $\mathrm{R}_{\mathrm{CY}}$ values are 0.98 and are indicative for a loss of FRET. As another control we tested a previously described interaction partner of DivIVA, wild type ParB, and a non-interacting point mutant, ParBR21A. (Donovan et al., 2012). RCY values were 1.16 for DivIVA-ParB interaction and 0.95 for DivIVA and ParBR21A (Figure S2; Table 3). Finally, when only YFP or YFP-tagged proteins were expressed, the emission spectra lack a CFP signal resulting in high $\mathrm{R}_{\mathrm{CY}}$ values ( $\left.>1.3\right)$.

\section{A POSITIVELY CHARGED AMINO ACID IN THE N-TERMINUS OF DivIVA IS CRUCIAL FOR INTERACTION WITH RodA}

We utilized the established FRET assay to map interaction sites between DivIVA and RodA. Therefore, we co-expressed the DivIVA truncation mutants with full length RodA in E. coli and evaluated the interaction microscopically (Figure 2). Here we show that DivIVA $\Delta$ C20 is not able to enrich RodA at the poles. DivIVA $\Delta$ C40 and CC2 both appear cytoplasmic and consequently do not interact with RodA. Interestingly, DivIVA $\Delta \mathrm{N}$ turned out to be unable to enrich RodA to the cell poles (Figure 2, forth column), thereby implicating the involvement of the N-terminal domain for RodA interaction. DivIVA $\Delta \mathrm{M}$ showed the same localization and interaction behavior as full length DivIVA (Figure 2, fifth column), thus suggesting that the middle domain is not involved in RodA interaction. We further analyzed the $\mathrm{N}$-terminal domain by mutating the lysine residue at position 20 (K20). When we mutated K20 to a phenylalanine, RodA enrichment was completely abolished (Figure 3, left column). The FRET ratio of 0.93 is in the range 
Table 3 | Classification and apportioning of FRET ratios into subgroups representing the interaction situation of the fusion proteins.

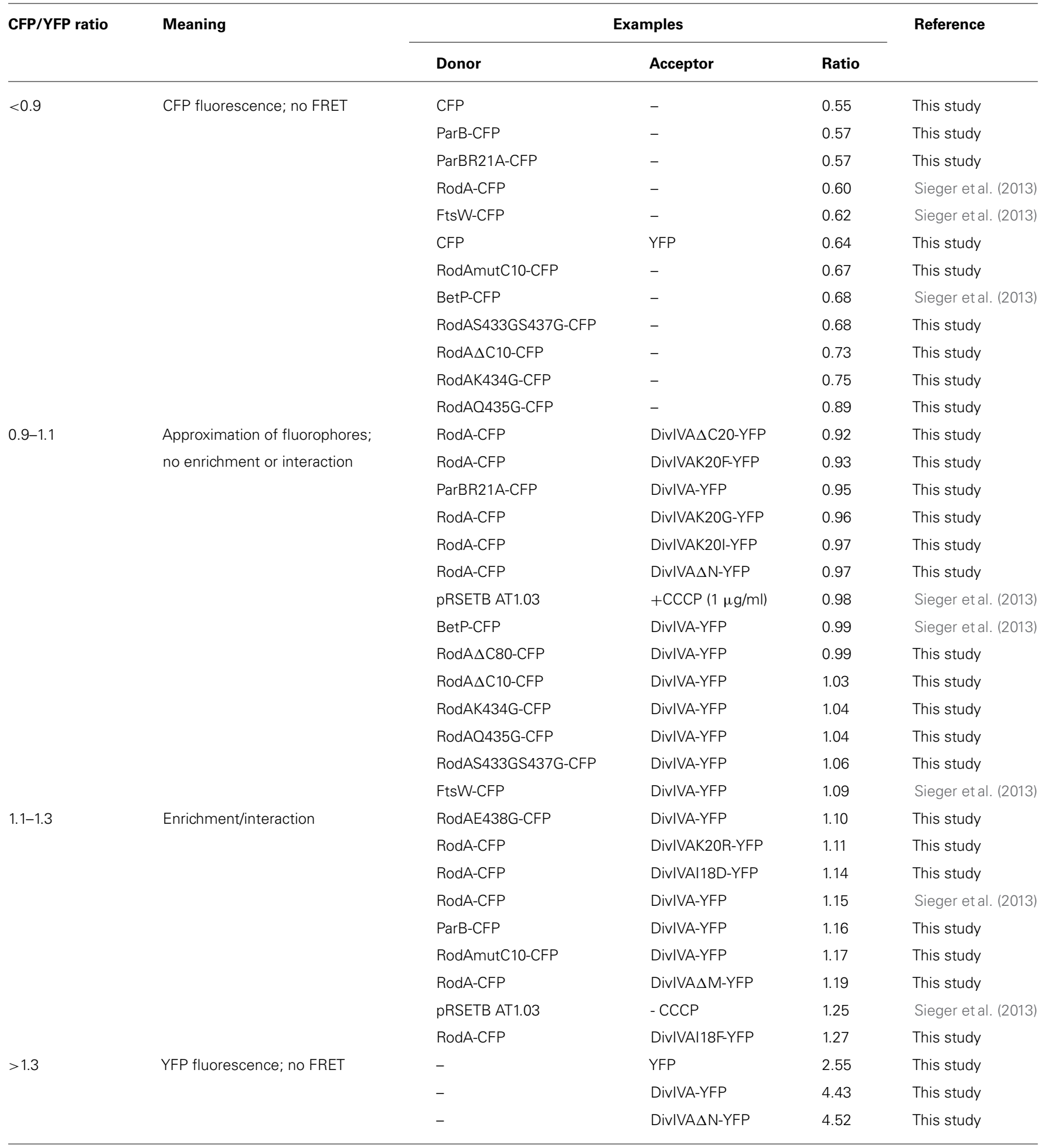

of approximation without interaction (Table 3). Similar, mutations K20I and K20G abolished interaction of DivIVA with RodA (Figure 3, middle columns). To address whether the observed loss of interaction depends on the positive charge, we constructed a conservative replacement, K20R. Interestingly, the co-localization of RodA was restored in the K20R mutant, implicating that a positive charge is necessary for interaction with RodA (Figure 3, right column, white arrows; $\mathrm{R}_{\mathrm{CY}}$ value of 1.11 ). DivIVA variants I18D 


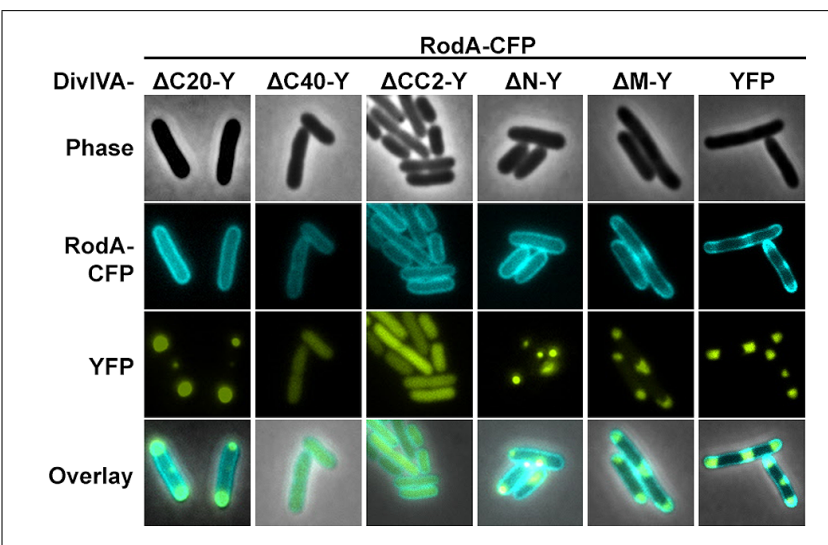

FIGURE 2 | Co-localization studies of DivIVA-YFP mutants with RodA-CFP from $\boldsymbol{C}$. glutamicum. DivIVA $\triangle$ C20-YFP is likely non-functional and thus cannot recruit RodA to the cell poles (first column).

DivIVA $\triangle$ C40-YFP and DivIVACC2-YFP, which appear cytoplasmic, cannot be interpreted in terms of co-localization with RodA (second and third column). DivIVA $\triangle$ N-YFP does not to recruit RodA to the cell poles (fourth column), unlike DivIVA $\triangle M-Y F P$ (fifth column), and full length DivIVA-YFP (sixth column).

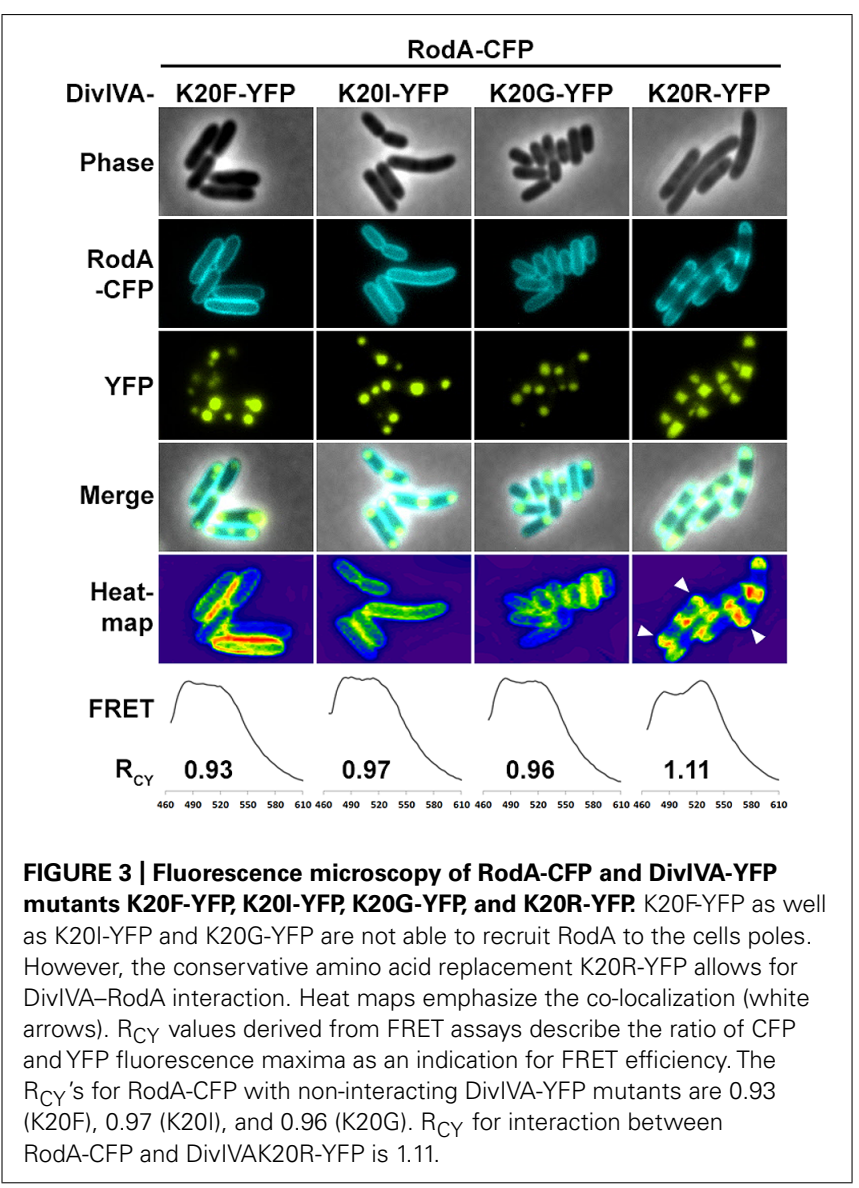

and $\mathrm{I} 18 \mathrm{~F}$ did not show any alteration in RodA interaction (Figure S3). Both mutants were able to co-localize RodA-CFP, supporting the notion that not all mutations in that region interfere with RodA interaction.

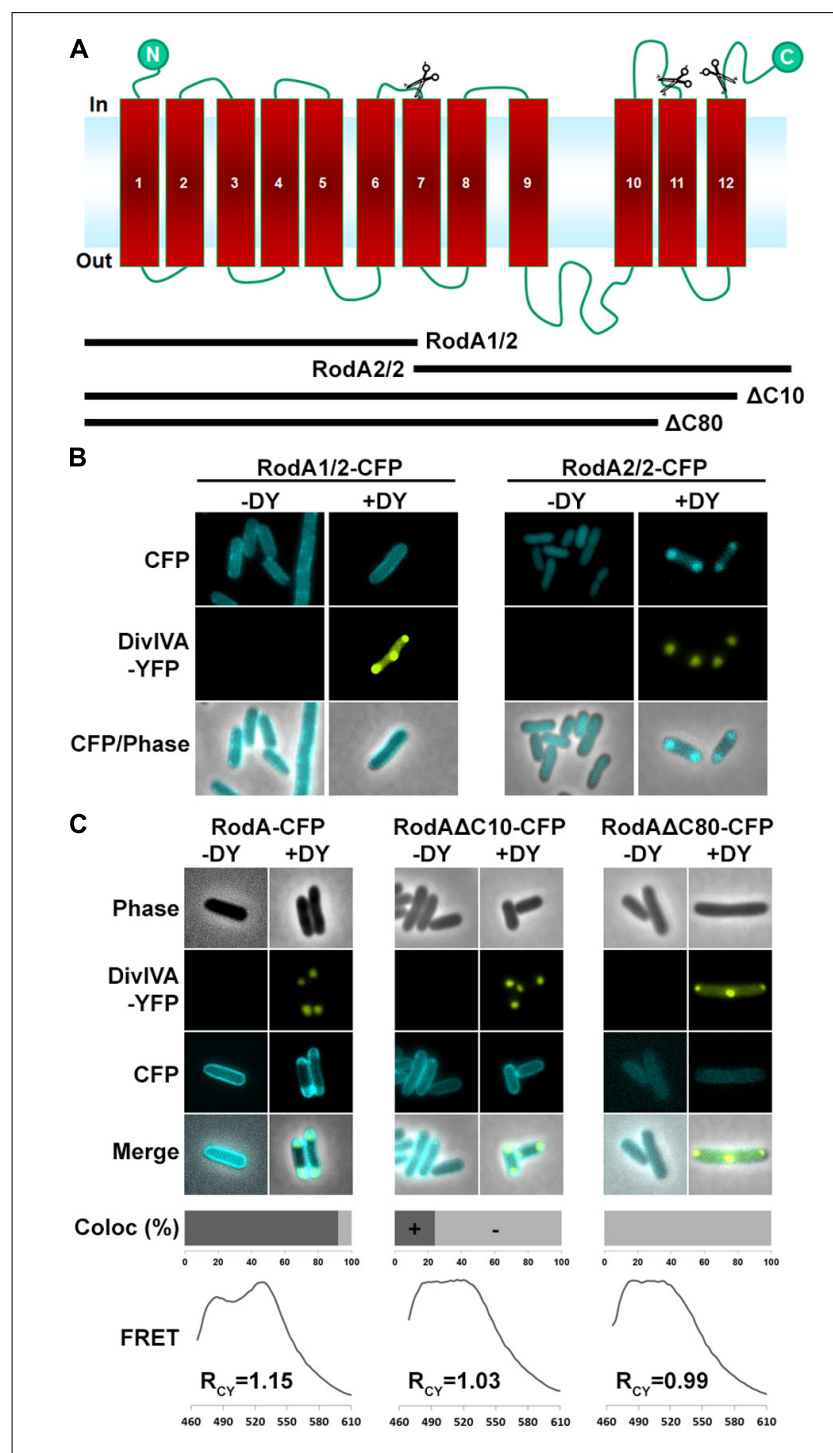

FIGURE 4 | (A) Topology model of RodA according to topology prediction (TMHMM). Scissors indicate truncation sites. The protein possesses 12 transmembrane domains and both ends are at the cytoplasmic site. (B) Fluorescence microscopy images of full length DivIVA-YFP and the two truncation mutants RodA1/2-CFP and RodA2/2-CFP. While individually expressed RodA1/2-CFP localizes to the membrane (first column), co-localization with DivIVA-YFP seems to be abolished (second column). RodA2/2-CFP lost completely its membrane localization and appears cytoplasmic (third column); however, when co-expressed with DivIVA, it is co-localized to DivIVA foci (forth column). (C) Localization of RodA-CFP, RodA $\Delta$ C10-CFP, and RodA $\triangle$ C80-CFP (-DY) and co-localization with DivIVA-YFP (+DY). Full length RodA co-localizes to $95 \%$ of all DivIVA foci, RodA $\triangle$ C10-CFP co-localizes to $20 \%$, and RodA $\triangle$ C80-CFP does not co-localize with DivIVA-YFP. FRET measurements confirm these observations. $R_{C Y}$ values are 1.15 for full length RodA, 1.03 for RodA $\Delta$ C10-CFP, and 0.99 for RodA $\Delta$ C80-CFP.

\section{RodA's C-TERMINUS IS INVOLVED IN INTERACTION WITH DivIVA}

Next we aimed to identify the RodA interaction site with DivIVA. Figure 4A shows a topology model of RodA according to a topology prediction simulation (TMHMM; Arnold et al., 2006). The protein harbors 12 transmembrane domains and both termini 
are facing the cytoplasm. To identify the interaction site with DivIVA we first divided the protein into two CFP-tagged halves and expressed them individually and together with DivIVA in E. coli (Figure 4B). It turned out that the N-terminal part (RodA1/2) localized to the membrane, however, it did not colocalize with DivIVA. The C-terminal part (RodA2/2) appeared cytoplasmic, but co-localized completely with DivIVA, implicating that the interaction site must be in the C-terminal half of the protein, although the truncated protein is apparently not inserted correctly into the membrane. We then made CFP-tagged truncations of 10 and 80 AAs from the C-terminus, ensuring cytoplasmic localization of the fluorophore. Whereas $>90 \%$ of full length RodA-CFP co-localized with DivIVA foci $\left(R_{\mathrm{CY}}=1.15\right)$, co-localization of RodA $\Delta$ C10-CFP was reduced to approximately $20 \%\left(R_{\mathrm{CY}}=1.03\right)$ and completely abolished for RodA $\Delta \mathrm{C} 80$ CFP $\left(R_{\mathrm{CY}}=0.99\right.$; Figure $\left.4 \mathrm{C}\right)$. Apparently, the C-terminal 10 AAs contribute to the RodA-DivIVA interaction. We finally tested several point mutations in the C-terminal domain of RodA. We reasoned that maybe a negatively charged residue might interact with K20 that we identified within DivIVA to be responsible for interaction. In spite of this, the variant RodAE438G did not abolish interaction $\left(R_{\mathrm{CY}}=1.10\right)$. RodAmut10C-CFP, a strain where all C-terminal 10 AAs of RodA were mutated into 10 AAs with similar residues $\left(\mathrm{K} \rightarrow \mathrm{R}, \mathrm{Q} \rightarrow \mathrm{N}, \mathrm{A} \rightarrow \mathrm{G}, R_{\mathrm{CY}}=1.17\right.$, Figure 5) preserved the interaction. However, point mutants K434G, Q435G, and the double mutant S433G-S437G decreased interaction with DivIVA $\left(R_{\mathrm{CY}}=1.04,1.04\right.$, and 1.05), implicating

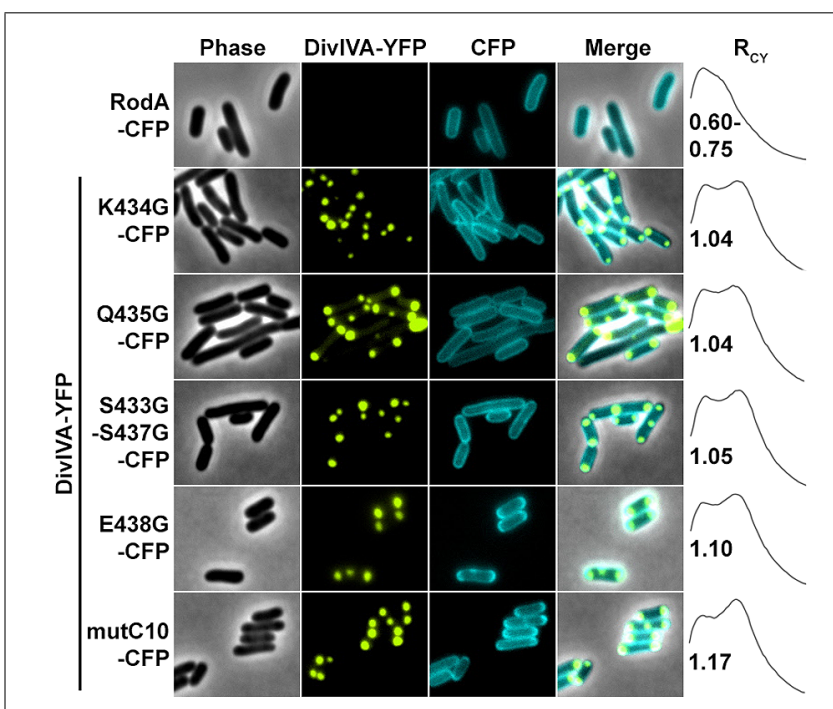

FIGURE 5 | Fluorescence microscopy images and $\mathbf{R}_{\mathrm{CY}}$ values of RodA-CFP mutants expressed with DivIVA-YFP. When expressed individually all RodA-CFP point mutants showed membrane localization identical to wild type RodA-CFP, as shown exemplarily in the first row. Co-expression and localization with DivIVA-YFP reveals effects of RodA mutants K434G, Q435G and the double mutant S433G/S437G, implicating that these four AAs are essential for interaction with DivIVA (rows 2-4). Point mutant E438G has no effect on co-localization (fifth row), as well as mutant mutC10 where the last $10 \mathrm{AAs}$ are changed to $10 \mathrm{AAs}$ with similar residues (WT sequence: MSKQASEVAA $\rightarrow$ AVRNGIADGG). These observations are confirmed by $\mathrm{R}_{C Y}$ measurements. A positive control with full-length proteins can be found in Figure 2 and (Sieger et al., 2013). an essential role of these four AAs in DivIVA-RodA interaction (Figure 5).

To support the data obtained with the heterologous expression system, we checked subcellular localization and growth complementation of RodA mutants in C. glutamicum. Therefore, we applied fluorescence microscopy after homologous expression of the truncation mutants in $\triangle \operatorname{rodA}$ (Figure 6A, strains BSC014-16). In addition we used a DivIVA-mCherry background as topological marker for the cell poles and septa. Whereas full length RodA-GFP localized to the cell poles in a DivIVA-dependent manner (yellow arrows; Sieger et al., 2013), RodA $\Delta$ C10-GFP localized only to some poles and not always co-localized with DivIVA. Instead, most of the RodA $\triangle$ C10-GFP formed random foci in the cell that did not co-localize with DivIVA (white arrows). These two observations corroborate the situation in E. coli, where only $20 \%$ of RodA $\triangle \mathrm{C} 10$ foci co-localized with DivIVA, implicating loss of tight interaction. RodA $\Delta$ C80-GFP appeared cytoplasmic and co-localization could not be observed, identical to the situation observed in E. coli. These observations were confirmed in a growth experiment on BHI-Agar plates (Figure 6B). Wild type and the complementation strain $\Delta \operatorname{rodA} /$ RodA-GFP grew normal, whereas $\Delta \operatorname{rodA} / \operatorname{RodA} \Delta \mathrm{C} 10$-GFP showed slight growth defects. All other strains $(\Delta \operatorname{rod} A, \Delta \operatorname{rodA} / \operatorname{RodA} \Delta \mathrm{C} 80-\mathrm{GFP}, \Delta \operatorname{rodA} / \operatorname{Rod} \mathrm{A} 1 / 2-\mathrm{GFP}$, $\Delta \operatorname{rodA} \operatorname{RodA} 2 / 2-\mathrm{GFP})$ were not able to support growth within $12 \mathrm{~h}$ of incubation at $30^{\circ} \mathrm{C}$.

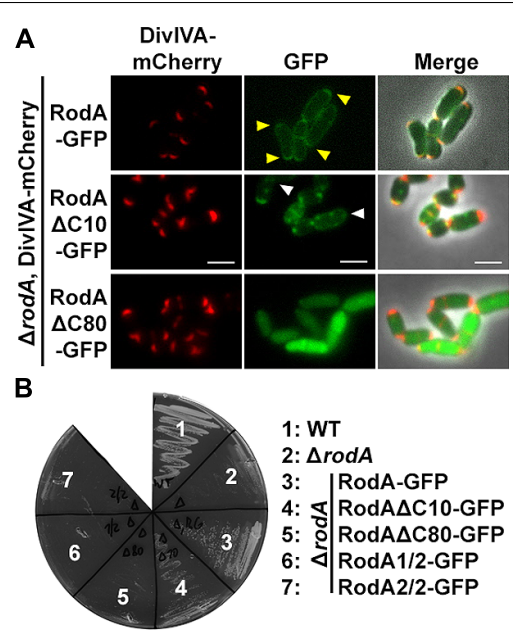

FIGURE 6 | (A) Fluorescence microscopy images of C. glutamicum DivIVA-mCherry, $\Delta$ rodA (BSC002), complemented with full length and truncated versions of RodA-GFP. RodA-GFP localizes DivIVA-like to the cell poles (first row, yellow arrows, strain BSC014). RodA $\triangle$ C10-GFP forms random foci in the cell and several DivIVA foci are free of RodA $\triangle$ C10-GFP, implicating reduced interaction (second row, white arrows, strain BSC015). RodA $\triangle$ C80-GFP appears cytoplasmic, polar foci are not observed (third row, strain BSC016). Fluorescence images were taken at equal exposure times (250 ms for mCherry and $500 \mathrm{~ms}$ for GFP). Scale bars: $2 \mu \mathrm{m}$.

(B) Growth experiment of WT, $\triangle$ rodA and complementation strains on Brain Heart Infusion agar. WT and full length complementation showed normal growth after $12 \mathrm{~h}$ at $30^{\circ} \mathrm{C}$ (Sieger et al., 2013). Complementation with RodA $\triangle$ C10-GFP (strain BSC015) had a slight growth defect, reflecting the observation of reduced DivIVA interaction. $\triangle$ rodA (BSC001) as well as complementation strains RodA $\triangle$ C80-GFP (BCS016), RodA1/2-GFP (BSC017), and RodA2/2-GFP (BSC018) could not develop colonies within $12 \mathrm{~h}$. 


\section{DISCUSSION}

Spatial and temporal organization is a major task in cell cycle regulation of all living species. Topological determinants like DivIVA are involved in spatial regulation of protein machineries such as the Min system in B. subtilis and Listeria monocytogenes (Bramkamp et al., 2008; Kaval et al., 2014) or the apical growth machinery in C. glutamicum (Letek et al., 2008; Sieger et al., 2013). Until now, it was unclear how the apical growth machinery is positioned in actinobacteria. Earlier studies suggested a link between DivIVA and penicillin-binding proteins (Letek et al., 2008), but recently, we could demonstrate the interaction between DivIVA and RodA in C. glutamicum (Sieger et al., 2013). However, the protein domains mediating interactions were not mapped. Here, we now identified the N-terminal domain of DivIVA as interaction partner of the C-terminal tail of RodA. We identified a positively charged residue (K20) in DivIVA to play a crucial role in this interaction. Loss of the positive charge at this position abolishes DivIVA-RodA interaction, while the conservative mutation, K20R, restores protein-protein interaction. The N-terminal domain of DivIVA is considered to be important for membranebinding. The structure of the B. subtilis DivIVA reveals that the $\mathrm{N}$-terminus folds into an intertwined-loop which exposes the hydrophobic residue F17. Mutational analysis shows that F17 is essential for membrane binding of the B. subtilis DivIVA. Moreover, the binding is backed by positive charged residues (R18; Oliva et al., 2010). Sequence alignments reveal that the N-terminal domain of DivIVA is highly homologous in most Gram positive bacteria. However, residue F17 from B. subtilis DivIVA is not conserved in actinobacteria. Streptomyces, Corynebacterium, and Mycobacterium species rather contain a positively charged residue at the corresponding site (Figure 1A). The actinobacterial DivIVA homologs contain sequence insertions and the hydrophobic residue mediating membrane association is thus likely I18 in case of C. glutamicum. Although mutation of such to an aspartate did not abolish membrane association compared to WT, localization of I18D in the heterologous host is slightly different compared to wild type (Figure 1C). Mutation of I18 to a phenylalanine, whose hydrophobic character is even more distinctive, restored polar localization of DivIVA and revealed wild type like membrane association. While membrane binding for the B. subtilis DivIVA has been studied in great detail, we know less about membrane binding properties of the actinobacterial proteins. Subcellular localization of C. glutamicum DivIVA shows some obvious differences compared to its B. subtilis homolog. While the B. subtilis DivIVA localizes tightly underneath the polar membrane (Edwards and Errington, 1997), forming a crescentlike structure lining the pole, the C. glutamicum protein localizes to the cell poles, but reaches into the cytoplasm (Letek et al., 2009; Donovan et al., 2012), forming a large complex, similar to the PopZ protein found in C. crescentus (Laloux and Jacobs-Wagner, 2013). It is therefore likely that membrane binding mechanisms are different in B. subtilis and C. glutamicum DivIVA proteins. Support to this notion comes from localization studies using truncated DivIVA (DivIVA $\Delta \mathrm{N}$ ), lacking the N-terminal domain. Although, DivIVA $\Delta \mathrm{N}$ is significantly more soluble compared to the full length protein, about $64 \%$ of the protein is still membrane associated. Despite its role in membrane interaction, the N-terminal domain of DivIVA is essential for interaction with RodA. The positive charge at residue $\mathrm{K} 20$ is required for interaction. It is plausible that the exposed, N-terminal loop of DivIVA is required for interaction with a membrane integral protein such as RodA. A central domain of DivIVA has been shown earlier to promote interaction with the origin-binding protein ParB (Donovan et al., 2012), indicating the modular character of DivIVA which encompasses various domains to mediate protein-protein interaction to various partner proteins.

RodA is an integral membrane protein with 12 predicted transmembrane helices. This topology gives rise to several putative binding-sites that could mediate DivIVA binding. A first, rough truncation study where we expressed RodA in two halves indicated that the DivIVA interaction site is likely situated in the C-terminal part of RodA. Further truncation analysis revealed that the last 10 AAs, forming the C-terminal domain facing the cytoplasm are contributing to the RodA-DivIVA interaction. RodA $\Delta \mathrm{C} 10$ has a drastically reduced FRET interaction with DivIVA; however, expression of RodA $\Delta \mathrm{C} 10$ in C. glutamicum to some extent complements growth in a $\Delta \operatorname{rod} A$ strain background. Only truncation of the last $80 \mathrm{AAs}$ from the C-terminus completely abolishes localization and interaction with DivIVA. A similar situation has been reported for FtsW, a RodA homolog that is involved in cell division. FtsW interacts via its C-terminal end with FtsZ during cytokinesis of $M$. tuberculosis (Datta et al., 2002). We were able to pinpoint amino acid residues responsible for RodA-DivIVA interaction in RodA (K434G, Q435G, and the double mutant S433G-S437G), suggesting that the RodA C-terminal domain forms an interaction domain which is built by several amino acid residues.

In earlier work we have been showing that ParB interacts with DivIVA in C. glutamicum. We have mapped the interaction sites and identified a central region in DivIVA (AAs 144-229) as interaction site with ParB (Donovan et al., 2012). Thus, DivIVA exhibits several exclusive interaction domains allowing DivIVA to act as interaction hub for the connection of apical cell growth and chromosome orientation. The N-terminal region of B. subtilis DivIVA interacts with MinJ, a multispan transmembrane protein involved in cytokinesis and division site selection (van Baarle et al., 2013). Thus, DivIVA proteins have evolved to contain interaction motifs for several protein-protein interactions. In actinobacteria DivIVA proteins are essential, likely because they are involved in the spatio-temporal control of two essential cellular processes, cell elongation, and chromosome segregation. Consistent with the fundamental role of DivIVA in these bacteria, actinobacterial DivIVA proteins are larger, containing sequence insertions, when compared to their firmicute counterparts. Future analysis might focus on the regulation of the various proteinprotein interactions. DivIVA has been identified as substrate of several protein kinases. Examples of DivIVA phosphorylation have been reported for M. tuberculosis (Kang et al., 2005), Streptomyces coelicolor (Hempel et al., 2012), and Streptococcus pneumoniae (Beilharz et al., 2012). Thereby, phosphorylation of DivIVA has major implications in cell growth or division (Fleurie et al., 2012). It is likely possible that a similar regulatory mechanisms determines chromosome segregation and cell elongation in C. glutamicum. 


\section{ACKNOWLEDGMENTS}

The authors acknowledge financial support from the Deutsche Forschungsgemeinschaft (BR2915/6-1) and the Federal ministry of Education and Research (BMBF, 031A302B). We thank Karin Schubert for critical comments on the manuscript.

\section{SUPPLEMENTARY MATERIAL}

The Supplementary Material for this article can be found online at: http://www.frontiersin.org/journal/10.3389/fmicb.2014.00738/ abstract

\section{REFERENCES}

Arnold, K., Bordoli, L., Kopp, J., and Schwede, T. (2006). The SWISS-MODEL workspace: a web-based environment for protein structure homology modelling. Bioinformatics 22, 195-201. doi: 10.1093/bioinformatics/bti770

Barh, D., Jain, N., Tiwari, S., Parida, B. P., D'afonseca, V., Li, L., et al. (2011). A novel comparative genomics analysis for common drug and vaccine targets in Corynebacterium pseudotuberculosis and other CMN Group of human pathogens. Chem. Biol. Drug Des. 78, 73-84. doi: 10.1111/j.1747-0285.2011. 01118.x

Beilharz, K., Nováková, L., Fadda, D., Branny, P., Massidda, O., and Veening, J.-W. (2012). Control of cell division in Streptococcus pneumoniae by the conserved Ser/Thr protein kinase StkP. Proc. Natl. Acad. Sci. U.S.A. 109, E905-E913. doi: 10.1073/pnas.1119172109

Ben-Yehuda, S., Rudner, D. Z., and Losick, R. (2003). RacA, a bacterial protein that anchors chromosomes to the cell poles. Science 299, 532-536. doi: 10.1126/science.1079914

Bramkamp, M., Emmins, R., Weston, L., Donovan, C., Daniel, R. A., and Errington, J. (2008). A novel component of the division-site selection system of Bacillus subtilis and a new mode of action for the division inhibitor MinCD. Mol. Microbiol. 70, 1556-1569. doi: 10.1111/j.1365-2958.2008.06501.x

Briley, K. Jr., Prepiak, P., Dias, M. J., Hahn, J., and Dubnau, D. (2011). Maf acts downstream of ComGA to arrest cell division in competent cells of B. subtilis. Mol. Microbiol. 81, 23-39. doi: 10.1111/j.1365-2958.2011.07695.x

Brown, P. J. B., Kysela, D. T., and Brun, Y. V. (2011). Polarity and the diversity of growth mechanisms in bacteria. Semin. Cell Dev. Biol. 22, 790-798. doi: 10.1016/j.semcdb.2011.06.006

Cayabyab, M. J., Macovei, L., and Campos-Neto, A. (2012). Current and novel approaches to vaccine development against tuberculosis. Front. Cell Infect. Microbiol. 2:154. doi: 10.3389/fcimb.2012.00154

Cowling, P., and Hall, L. (1993). Corynebacterium striatum: a clinically significant isolate from sputum in chronic obstructive airways disease. J. Infect. 26, 335-336. doi: 10.1016/0163-4453(93)95823-2

Datta, P., Dasgupta, A., Bhakta, S., and Basu, J. (2002). Interaction between FtsZ and FtsW of Mycobacterium tuberculosis. J. Biol. Chem. 277, 24983-24987. doi: 10.1074/jbc.M203847200

Donovan, C., and Bramkamp, M. (2014). Cell division in Corynebacterineae. Front. Microbiol. 5:132. doi: 10.3389/fmicb.2014.00132

Donovan, C., Sieger, B., Krämer, R., and Bramkamp, M. (2012). A synthetic Escherichia coli system identifies a conserved origin tethering factor in Actinobacteria. Mol. Microbiol. 84, 105-116. doi: 10.1111/j.1365-2958.2012. 08011.x

dos Santos, V. T., Bisson-Filho, A. W., and Gueiros-Filho, F. J. (2012). DivIVAmediated polar localization of ComN, a posttranscriptional regulator of Bacillus subtilis. J. Bacteriol. 194, 3661-3669. doi: 10.1128/JB.05879-11

Edwards, D. H., and Errington, J. (1997). The Bacillus subtilis DivIVA protein targets to the division septum and controls the site specificity of cell division. Mol. Microbiol. 24, 905-915. doi: 10.1046/j.1365-2958.1997.3811764.x

Eikmanns, B. J., Kleinertz, E., Liebl, W., and Sahm, H. (1991). A family of Corynebacterium glutamicum/Escherichia coli shuttle vectors for cloning, controlled gene expression, and promoter probing. Gene 102, 93-98. doi: 10.1016/0378-1119(91)90545-M

Eswaramoorthy, P., Winter, P. W., Wawrzusin, P., York, A. G., Shroff, H., and Ramamurthi, K. S. (2014). Asymmetric division and differential gene expression during a bacterial developmental program requires DivIVA. PLoS Genet. 10:e1004526. doi: 10.1371/journal.pgen.1004526
Fleurie, A., Cluzel, C., Guiral, S., Freton, C., Galisson, F., Zanella-Cleon, I., et al. (2012). Mutational dissection of the S/T-kinase StkP reveals crucial roles in cell division of Streptococcus pneumoniae. Mol. Microbiol. 83, 746-758. doi: 10.1111/j.1365-2958.2011.07962.x

Hempel, A. M., Cantlay, S., Molle, V., Wang, S.-B., Naldrett, M. J., Parker, J. L., et al. (2012). The Ser/Thr protein kinase AfsK regulates polar growth and hyphal branching in the filamentous bacteria Streptomyces. Proc. Natl. Acad. Sci. U.S.A. 109, E2371-E2379. doi: 10.1073/pnas.1207409109

Henriques, A. O., Glaser, P., Piggot, P. J., and Moran, C. P. Jr. (1998). Control of cell shape and elongation by the rodA gene in Bacillus subtilis. Mol. Microbiol. 28, 235-247. doi: 10.1046/j.1365-2958.1998.00766.x

Ikeda, M., and Nakagawa, S. (2003). The Corynebacterium glutamicum genome: features and impacts on biotechnological processes. Appl. Microbiol. Biotechnol. 62, 99-109. doi: 10.1007/s00253-003-1328-1

Imamura, H., Nhat, K. P., Togawa, H., Saito, K., Iino, R., Kato-Yamada, Y., et al. (2009). Visualization of ATP levels inside single living cells with fluorescence resonance energy transfer-based genetically encoded indicators. Proc. Natl. Acad. Sci. U.S.A. 106, 15651-15656. doi: 10.1073/pnas.0904764106

Jones, L. J. F., Carballido-López, R., and Errington, J. (2001). Control of cell shape in bacteria: helical, actin-like filaments in Bacillus subtilis. Cell 104, 913-922. doi: 10.1016/S0092-8674(01)00287-2

Kang, C. M., Abbott, D. W., Park, S. T., Dascher, C. C., Cantley, L. C., and Husson, R. N. (2005). The Mycobacterium tuberculosis serine/threonine kinases PknA and PknB: substrate identification and regulation of cell shape. Genes Dev. 19, 16921704. doi: 10.1101/gad.1311105

Kaval, K. G., Rismondo, J., and Halbedel, S. (2014). A function of DivIVA in Listeria monocytogenes division site selection. Mol. Microbiol. 94, 637-654. doi: $10.1111 / \mathrm{mmi} .12784$

Kruse, T., Bork-Jensen, J., and Gerdes, K. (2005). The morphogenetic MreBCD proteins of Escherichia coli form an essential membrane-bound complex. Mol. Microbiol. 55, 78-89. doi: 10.1111/j.1365-2958.2004.04367.x

Laloux, G., and Jacobs-Wagner, C. (2013). Spatiotemporal control of PopZ localization through cell cycle-coupled multimerization. J. Cell Biol. 201, 827-841. doi: $10.1083 /$ jcb. 201303036

Laloux, G., and Jacobs-Wagner, C. (2014). How do bacteria localize proteins to the cell pole? J. Cell Sci. 127, 11-19. doi: 10.1242/jcs.138628

Lenarcic, R., Halbedel, S., Visser, L., Shaw, M., Wu, L. J., Errington, J., et al. (2009). Localisation of DivIVA by targeting to negatively curved membranes. EMBO J. 28, 2272-2282. doi: 10.1038/emboj.2009.129

Letek, M., Fiuza, M., Ordonez, E., Villadangos, A. F., Flardh, K., Mateos, L. M., et al. (2009). DivIVA uses an N-terminal conserved region and two coiled-coil domains to localize and sustain the polar growth in Corynebacterium glutamicum. FEMS Microbiol. Lett. 297, 110-116. doi: 10.1111/j.1574-6968.2009. 01679.x

Letek, M., Ordonez, E., Vaquera, J., Margolin, W., Flardh, K., Mateos, L. M., et al. (2008). DivIVA is required for polar growth in the MreB-lacking rod-shaped actinomycete Corynebacterium glutamicum. J. Bacteriol. 190, 3283-3292. doi: 10.1128/JB.01934-07

Lin, L., and Thanbichler, M. (2013). Nucleotide-independent cytoskeletal scaffolds in bacteria. Cytoskeleton 70, 409-423. doi: $10.1002 / \mathrm{cm} .21126$

Locci, R., and Schaal, K. P. (1980). Apical growth in facultative anaerobic actinomycetes as determined by immunofluorescent labeling. Zentralbl. Bakteriol. A. 246, 112-118. doi: 10.1016/S0172-5599(80)80102-7

Mohammadi, T., Van Dam, V., Sijbrandi, R., Vernet, T., Zapun, A., Bouhss, A., et al. (2011). Identification of FtsW as a transporter of lipid-linked cell wall precursors across the membrane. EMBO J. 30, 1425-1432. doi: 10.1038/emboj. 2011.61

Muchova, K., Kutejova, E., Scott, D. J., Brannigan, J. A., Lewis, R. J., Wilkinson, A. J., et al. (2002). Oligomerization of the Bacillus subtilis division protein DivIVA. Microbiology 148, 807-813.

Noirclerc-Savoye, M., Morlot, C., Gerard, P., Vernet, T., and Zapun, A. (2003). Expression and purification of FtsW and RodA from Streptococcus pneumoniae, two membrane proteins involved in cell division and cell growth, respectively. Protein Expr. Purif. 30, 18-25. doi: 10.1016/S1046-5928(03) 00051-2

Oliva, M. A., Halbedel, S., Freund, S. M., Dutow, P., Leonard, T. A., Veprintsev, D. B., et al. (2010). Features critical for membrane binding revealed by DivIVA crystal structure. EMBO J. 29, 1988-2001. doi: 10.1038/emboj.2010.99 
Pastoret, S., Fraipont, C., Den Blaauwen, T., Wolf, B., Aarsman, M. E. G., Piette, A., et al. (2004). Functional analysis of the cell division protein FtsW of Escherichia coli. J. Bacteriol. 186, 8370-8379. doi: 10.1128/jb.186.24.8370-83 79.2004

Patrick, J. E., and Kearns, D. B. (2008). MinJ (YvjD) is a topological determinant of cell division in Bacillus subtilis. Mol. Microbiol. 70, 1166-1179. doi: 10.1111/j.1365-2958.2008.06469.x

Popham, D. L., and Young, K. D. (2003). Role of penicillin-binding proteins in bacterial cell morphogenesis. Curr. Opin. Microbiol. 6, 594-599. doi: 10.1016/j.mib.2003.10.002

Real, G., Fay, A., Eldar, A., Pinto, S. M., Henriques, A. O., and Dworkin, J. (2008). Determinants for the subcellular localization and function of a nonessential SEDS protein. J. Bacteriol. 190, 363-376. doi: 10.1128/jb.01482-07

Sham, L. T., Butler, E. K., Lebar, M. D., Kahne, D., Bernhardt, T. G., and Ruiz, N. (2014). Bacterial cell wall. MurJ is the flippase of lipid-linked precursors for peptidoglycan biogenesis. Science 345, 220-222. doi: 10.1126/science.1254522

Sieger, B., Schubert, K., Donovan, C., and Bramkamp, M. (2013). The lipid II flippase RodA determines morphology and growth in Corynebacterium glutamicum. Mol. Microbiol. 90, 966-982. doi: 10.1111/mmi.12411

Stahlberg, H., Kutejova, E., Muchova, K., Gregorini, M., Lustig, A., Muller, S. A., et al. (2004). Oligomeric structure of the Bacillus subtilis cell division protein DivIVA determined by transmission electron microscopy. Mol. Microbiol. 52, 1281-1290. doi: 10.1111/j.1365-2958.2004.04074.x

Tauch, A., Kirchner, O., Loffler, B., Gotker, S., Puhler, A., and Kalinowski, J. (2002). Efficient electrotransformation of Corynebacterium diphtheriae with a mini-replicon derived from the Corynebacterium glutamicum plasmid pGA1. Curr. Microbiol. 45, 362-367. doi: 10.1007/s00284-002-3728-3 van Baarle, S., Celik, I. N., Kaval, K. G., Bramkamp, M., Hamoen, L. W., and Halbedel, S. (2013). Protein-protein interaction domains of Bacillus subtilis DivIVA. J. Bacteriol. 195, 1012-1021. doi: 10.1128/JB.02171-12

Wu, L. J., and Errington, J. (2003). RacA and the Soj-Spo0J system combine to effect polar chromosome segregation in sporulating Bacillus subtilis. Mol. Microbiol. 49, 1463-1475. doi: 10.1046/j.1365-2958.2003.03643.x

Zeeuwen, P. L., Kleerebezem, M., Timmerman, H. M., and Schalkwijk, J. (2013). Microbiome and skin diseases. Curr. Opin. Allergy Clin. Immunol. 13, 514-520. doi: 10.1097/ACI.0b013e328364ebeb

Conflict of Interest Statement: The authors declare that the research was conducted in the absence of any commercial or financial relationships that could be construed as a potential conflict of interest.

Received: 15 October 2014; accepted: 05 December 2014; published online: 07 January 2015.

Citation: Sieger B and Bramkamp M (2015) Interaction sites of DivIVA and RodA from Corynebacterium glutamicum. Front. Microbiol. 5:738. doi: 10.3389/fmicb. 2014.00738

This article was submitted to Microbial Physiology and Metabolism, a section of the journal Frontiers in Microbiology.

Copyright (C) 2015 Sieger and Bramkamp. This is an open-access article distributed under the terms of the Creative Commons Attribution License (CC BY). The use, distribution or reproduction in other forums is permitted, provided the original author(s) or licensor are credited and that the original publication in this journal is cited, in accordance with accepted academic practice. No use, distribution or reproduction is permitted which does not comply with these terms. 